\title{
Ground-state energy and Wigner crystallization in thick 2D-electron systems
}

\author{
D. Jost* \\ Ecole Normale Supérieure de Lyon, 46 Allée d'Italie 69364 Lyon, France \\ M. W. C. Dharma-wardand \\ Institute of Microstructural Sciences, National Research Council of Canada, Ottawa, Canada. K1A OR6
}

(Dated: July 17, 2018)

\begin{abstract}
The ground state energy of the 2-D Wigner crystal is determined as a function of the thickness of the electron layer and the crystal structure. The method of evaluating the exchange-correlation energy is tested using known results for the infinitely-thin 2D system. Two methods, one based on the local-density approximation (LDA), and another based on the constant-density approximation (CDA) are established by comparing with quantum Monte-Carlo (QMC) results. The LDA and CDA estimates for the Wigner transition of the perfect $2 \mathrm{D}$ fluid are at $r_{s}=38$ and 32 respectively, compared with $r_{s}=35 \pm 5$ from QMC. For thick-2D layers as found in Hetero-junction-insulatedgate field-effect transistors, the LDA and CDA predictions of the Wigner transition are at $r_{s}=20.5$ and 15.5 respectively. Impurity effects are not considered here.
\end{abstract}

PACS numbers: PACS Numbers: 05.30.Fk, 71.10.-w, 71.45.Gm, 71.15.Mb

\section{INTRODUCTION}

Two-dimensional (2D) electron layers exist, for example, at the interface between GaAs and $\mathrm{Ga}_{1-x} \mathrm{Al}_{x}$ As, or at the interface of a metal oxide and a semiconductor. Such interfaces are important in field-effect transistors and other devices. The state of this 2D electrons system can be fluid or, at sufficiently low density, the electrons condense and crystallize 1, 2]. We define the 2D-density parameter $r_{s}$ given by $A / N=\pi r_{s}{ }^{2}$ where $A$ is the area occupied by the $N$ electrons. The Wigner solid appears for $r_{s} \geq 35 a_{0}[\underline{3}]$ where $a_{0}=\hbar^{2} /\left(m^{*} e^{* 2}\right)$ is the Bohr radius. Here $m^{*}, e^{*}$ are effective parameters for the mass and the charge of the electron, and absorb the dielectric constant, the band mass and other material properties of the system. Thus in GaAs, the effective atomic unit of energy is reduced from $27.21 \mathrm{eV}$ to the milli-Volt range. These (reduced) atomic units, such that $m^{*}=e^{*}=\hbar=1$ will be assumed through out this paper. There has been many studies of 2D-electron liquids or Wigner crystals [3, 4, [5, 6, 7, 8], especially using quantum Monte Carlo (QMC) simulations and other methods [, 9] assuming that the 2-D layers are infinitely thin. However, although the 2D electrons reside in the $(x, y)$-plane, they have a transverse density $\eta(z)$ confined to the lowest subband of the hetero-structure [1]. While the quasi-2D electron liquid has recently seen much attention, both experimental 11, 12] and theoretical 10, 13, 14, 15], the Wigner crystal in thick 2D layers has not been followed up since the work of Fujiki and Geldart [16]. Fujiki et al., have determined the effect of the 2D-layer thickness on the electrostatic energy and found that the hexagonal lattice is the most-stable crystal structure, as with the

\footnotetext{
*Email: daniel.jost@ens-lyon.fr

${ }^{\dagger}$ Email for correspondence: chandre.dharma-wardana@nrc.ca
}

$\delta$-thin 2D layer $(\delta 2 \mathrm{D})$. However, they did not consider the effect of exchange and correlation which is usually addressed via Quantum Monte Carlo methods, or via a detailed analysis of the correlated phonons in the electron crystal [7]. We note that recent Hartree-Fock (HF) calculations of $\delta 2 \mathrm{D}$ Wigner crystals using large plane-wave basis sets, e.g, those of Trail et al [17]., seem to recover a HF energy nearly identical to the single- Gaussian harmonic approximation for localized electrons. Various aspects of such a model have been considered in a brief but insightful paper by Nagy [18, 19]. In this study we show that the single-Gaussian approximation, and the local-density approximation (LDA), can recover the QMC total energy with surprising accuracy. We also show that a method based on constructing a constant-density approximation (CDA) to the inhomogeneous density [10, 20] can be profitably used for calculating the electrostatic potentials and the exchange-correlation energies of these systems.

The plan of the paper is as follows. In section 1 we introduce the Hamiltonian, the effective Coulomb interaction in quasi-2D layers, and calculate the electrostatic energy of the lattice for several 2D-crystal structures. Here we use the CDA to replace Fang-Howard type densities in the $z$-direction [1, 10], thus simplifying the analytical work. The details of lattice-sum evaluations are relegated to an appendix. In section III we consider the $\delta$-thin 2D layer and present results for the gaussian-localized model. we also present the exchange-correlation energy $E_{x c}$ calculation using the CDA and the LDA. The resulting total energy is very close to that of QMC and recovers a liquid $\rightarrow$ solid Wigner transition (WT) at $r_{s} \sim 32$ to 38 , while the current QMC estimate is $r_{s}=35 \pm 5$. In section IV we consider Gaussian-localized 2D systems with finite thickness, for 2D layers found in HIGFETS. That is, in systems where the layer thickness is also defined by the sheet density, as in Tan et al [11, 12]. Here we have no QMC results for comparison. The total energy of the quasi-2D Wigner crystal is compared with the total en- 
ergy of the quasi-2D liquid [10]. Here the WT is found to occur at $r_{s} \sim 15$ to 21 in quasi-2D layers realized in clean HIGFETS,

\section{THE COULOMB ENERGIES OF 2D LATTICES}

The Hamiltonian of our system is, in atomic units,

$$
H=H_{k e}+H_{e e}+H_{e b}+H_{b b}
$$

where the first term is the kinetic energy of the electrons. The three remaining terms are the electronelectron interaction and the interactions involving the uniform, static neutralizing background, indicated by the subscript $b$. This neutralizing background arises from a homogeneous layer of donor ions which have acquired a positive charge after donating their valence electrons in forming the 2D-electron layer. We assume that the electron layer is confined near the plane $z=0$ and extends into the region of $z>0$ due to the finite width of the envelope function. The donor ions are modeled by a homogeneous layer of positive charge of areal density $\rho_{d}=N / A$, situated at $z=-b_{d}$, where $b_{d}=\left|b_{d}\right|$ is a positive quantity. The $z$-direction density is $\eta(z)$, and in the plane, an areal density $\rho_{e}(\mathbf{r})$ with $\mathbf{r}=(x, y)$. The subband distribution $\eta(z)$ is usually modeled by a FangHoward distribution $\eta_{f h}(z)=\left(1 / 2 b^{3}\right) z^{2} e^{-\frac{z}{b}}$ (n.b., our $b=1 / b$ used in Ref. [1]), or various other forms, e.g, that of a quantum well. The form of the density is obtained by fitting to a self-consistent calculation of the Schrodinger equation for the electron motion in the $z$-direction. In our work, we do not repeat this calculation, but simply take the value of the parameter $b$, or other parameters needed to define the self-consistent solution for the subband. Moreover, as discussed below, such inhomogeneous densities can be replaced by a constant-density slab having an equivalent electrostatic potential, using the CDA discussed by Dharma-wardana 10. The CDA method [10] involves replacing an inhomogeneous density $\eta(z)$ by a slab of constant-density $\bar{\eta}$ of width $a$ linked to $\eta(z)$ by

$$
\bar{\eta}=\frac{1}{a}=\int \eta(z)^{2} d z
$$

This equation has also been proposed by Gori-Giorgi et al [20]., in a method for calculating system-adapted correlation energies. Using Eq. 2]a Fang-Howard (FH) density of length scale $b$ can be replace by a homogeneous density of width $a=(16 b) / 3$.

Consider two electrons in a quasi-2D layer separated by a distance $r$ in the 2D plane, and located at $z_{1}$ and $z_{2}$, with a $\mathrm{FH}$ distribution $\eta(z)$ in the $z$ direction. Then the Coulomb interaction is of the form

$$
W(r)=\int d z_{1} d z_{2} \frac{\eta\left(z_{1}\right) \eta\left(z_{2}\right)}{\left|r^{2}+\left(z_{1}-z_{2}\right)^{2}\right|^{1 / 2}}
$$

$W(r)$ may be written as $F(r) / r$ where $F(r)$ is the form factor. No analytic form exists if $\eta(z)$ is the $\mathrm{FH}$ form,

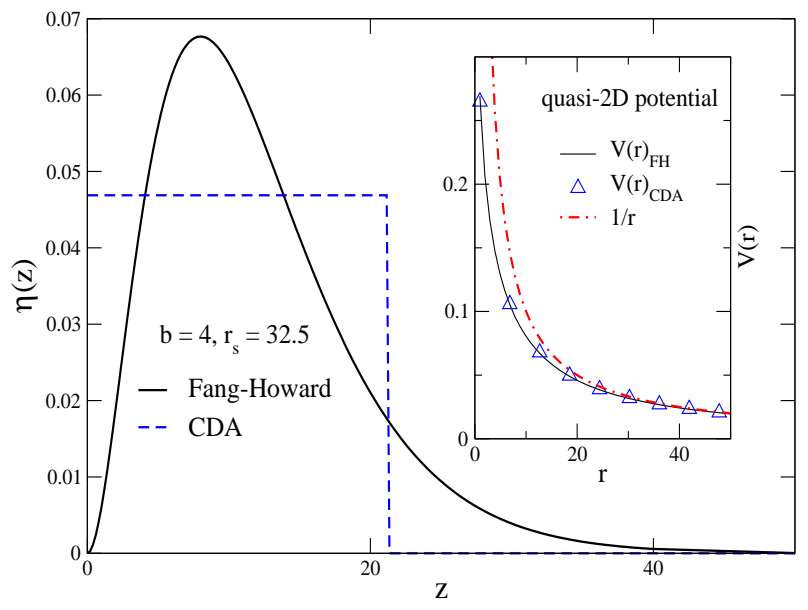

FIG. 1: (Color online) Profiles of the Fang-Howard density (solid curve) for $b=4$ and its equivalent constant density approximation(CDA, dashed curve). Inset: the bare Coulomb potential $1 / r$ and the Coulomb potential $F(r) / r$ modifed by the Fang-Howard profile. The triangles are calculated using the CDA. The CDA width $\mathrm{a}=21.33$ for $b=4$ corresponds to a HIGFET at $r_{s}=32.496$.

while the $q$-space form, $F(q) 2 \pi / q$ is analytically available. For GaAs/AlAs based HIGFET-like systems, it takes the form,

$$
F(q)=\left[1+\frac{9 q}{8 b^{\prime}}+\frac{3 q^{2}}{8 b^{\prime 2}}\right]\left[1+\frac{q}{b^{\prime}}\right]^{-2}
$$

where $b^{\prime}=1 / b$ and follows the definition in Ref. 11]. However, if the $\mathrm{FH}$ distribution is replaced by the CDA, then $F(r)$ and $F(q)$ are given by

$$
\begin{aligned}
W(r) & =V(r) F(s), \quad s=r / a, t=\sqrt{ }\left(1+s^{2}\right) \\
F(s) & =2 s\left[\log \frac{1-t}{s}+1-t\right]
\end{aligned}
$$

and

$$
\begin{aligned}
W(q) & =V(q) F(p), \quad p=q a, V(k)=2 \pi / q \\
F(p) & =(2 / p)\left\{\left(e^{-p}-1\right) / p+1\right\}
\end{aligned}
$$

The form factors $F(r), F(q)$ are a measure of the reduction of the strength of the 2D interaction due to the thickness effect. These results provide equivalent analytical formulae for the FH-density, and tend to the ideal 2D Coulomb potential when the width $a$ tends to zero. Also, for HIGFETS, it is known that the FH-parameter $b$ is linked to the $2 \mathrm{D}$ density parameter $r_{s}$. Hence it can be shown [10] that

$$
\begin{aligned}
b & =\left(2 r_{s}^{2} / 33\right)^{1 / 3} \\
a & =16 b / 3 \\
\beta & =b / r_{s}=\left[2 /\left(33 r_{s}\right)\right]^{1 / 3}
\end{aligned}
$$

Hence $\beta$, the $\mathrm{FH}$ parameter $b$ in units of $r_{s}$, and also the ratio $a / r_{s}$, i.e., ( $z$-width) $/(2 \mathrm{D}$-disk radius) decrease as $r_{s}^{1 / 3}$ with increasing $r_{s}$. 


\section{A. Coulomb energy}

In the following we do not at first specify the form of the transverse density $\eta(z)$. In calculating the Coulomb energy $E_{C o u}$, i.e, the electrostatic energy, we isolate the long-range contributions which cancel in the $q=0$ limit, since we are dealing with a homogeneous, neutralizing, static background. The total Coulomb energy is the sum, where

$$
E_{d d}(q)=\frac{1}{2} \int d^{2} r \int d^{2} r^{\prime} \rho_{d}^{2} \frac{e^{i \mathbf{q} \cdot\left(\mathbf{r}-\mathbf{r}^{\prime}\right)}}{\left|\mathbf{r}-\mathbf{r}^{\prime}\right|}
$$

$$
E_{C o u}=\lim _{q \rightarrow 0}\left[E_{d d}(q)+E_{e e}(q)+2 E_{e d}(q)\right]
$$

$$
\begin{aligned}
& E_{e e}(q)=\frac{1}{2} \int d^{2} r \int d^{2} r^{\prime} \rho_{e}(\mathbf{r}) \rho_{e}\left(\mathbf{r}^{\prime}\right) e^{i \mathbf{q} \cdot\left(\mathbf{r}-\mathbf{r}^{\prime}\right)} \int_{0}^{\infty} d z \int_{0}^{\infty} d z^{\prime} \frac{\eta(z) \eta\left(z^{\prime}\right)}{\left[\left(\mathbf{r}-\mathbf{r}^{\prime}\right)^{2}+\left(z-z^{\prime}\right)^{2}\right]^{1 / 2}} \\
& E_{e d}(q)=-\frac{1}{2} \int d^{2} r \int d^{2} r^{\prime} \rho_{d} \rho_{e}\left(\mathbf{r}^{\prime}\right) e^{i \mathbf{q} \cdot\left(\mathbf{r}-\mathbf{r}^{\prime}\right)} \int_{0}^{\infty} d z^{\prime} \frac{\eta\left(z^{\prime}\right)}{\left[\left(\mathbf{r}-\mathbf{r}^{\prime}\right)^{2}+\left(b_{d}+z^{\prime}\right)^{2}\right]^{1 / 2}}
\end{aligned}
$$

$E_{d d}$ is the interaction energy of the ions, $E_{e e}$ is the interaction of the electron layer and $E_{e d}$ is the energy due to interaction between the ions and the electrons. To calculate these terms, we proceed as in Fujiki and Geldart [16, 21].

$$
E_{d d}(q)=\frac{\pi A \rho_{d}^{2}}{q}=\frac{N}{q r_{s}^{2}}
$$

We introduce the integral transformation

$$
\frac{1}{|\mathbf{v}|}=\int_{0}^{\infty} \frac{d y}{\sqrt{\pi}} y^{-1 / 2} e^{-y|\mathbf{v}|^{2}}
$$

and note that

$$
\begin{aligned}
E_{e d}(q)= & -\frac{1}{2} \int d^{2} r \int d^{2} r^{\prime} \rho_{d} \rho_{e}\left(\mathbf{r}^{\prime}\right) e^{i \mathbf{q} \cdot\left(\mathbf{r}-\mathbf{r}^{\prime}\right)} \int_{0}^{\infty} d z^{\prime} \eta\left(z^{\prime}\right) \\
& \int_{0}^{\infty} \frac{d y}{\sqrt{\pi}} y^{-1 / 2} e^{-y\left|\mathbf{r}-\mathbf{r}^{\prime}\right|^{2}-y\left|b_{d}+z^{\prime}\right|^{2}} \\
= & -\frac{N}{2 \sqrt{\pi} r_{s}^{2}} \int_{0}^{\infty} d y y^{-3 / 2} e^{-\frac{q^{2}}{4 y}} \\
& \int_{0}^{\infty} d z^{\prime} \eta\left(z^{\prime}\right) e^{-y\left|z^{\prime}+b_{d}\right|^{2}}
\end{aligned}
$$

For $E_{e e}$, we use a lattice sum technique based on the $\theta$ Jacobi function, Eq. (19), and its imaginary transform, Eq.(20), given below:

$$
\begin{aligned}
& \theta(z, X) \equiv \sum_{l=-\infty}^{\infty} e^{2 \pi l z} e^{-\pi l^{2} X} \\
& \theta(z, X)=\frac{e^{\pi z^{2} / X}}{\sqrt{X}} \theta\left(\frac{z}{i X}, \frac{1}{X}\right)
\end{aligned}
$$

We decompose the lattice into rectangular sublattices indicated with sublattice vectors $\rho_{\mathbf{j}}$. So, the position vectors of the electrons on nodes $I$ and $J$ are given by

$$
\mathbf{r}_{\mathbf{I}}=m a_{1} \hat{x}+n a_{2} \hat{y}, \quad \mathbf{r}_{\mathbf{J}}=\left(m^{\prime} a_{1}+\rho_{j}^{x}\right) \hat{x}+\left(n^{\prime} a_{2}+\rho_{j}^{y}\right) \hat{y}
$$

where $m, m^{\prime}, n, n^{\prime}$ are integers, $a_{1}, a_{2}$ are lattice constants of sublattices. For example, in a square lattice $a_{1}=a_{2}$ and $\left\{\rho_{\mathbf{j}}\right\}=\{(0 ; 0)\}$, in a hexagonal lattice $a_{2}=\sqrt{3} a_{1}$ and $\left\{\rho_{\mathbf{j}}\right\}=\left\{(0 ; 0),\left(\frac{a_{1}}{2} ; \frac{a_{1} \sqrt{3}}{2}\right)\right\}$. To proceed further, we need to specify the form of the density. If the electrons are assumed to be exactly localized on the nodes of the crystal, then

$$
\rho_{e \delta}(\mathbf{r})=\sum_{I} \delta\left(\mathbf{r}-\mathbf{r}_{\mathbf{I}}\right) .
$$

Such exact localization of the electrons provides the model for the classical electrostatic energy, i.e, the Madelung energy. In the quantum calculation we suppose that each electron is localized around a node $I$ of the lattice and the wavefunction is taken to be a gaussian normalized over the 2D plane,

$$
\phi_{I}(\mathbf{r})=\sqrt{\frac{2 \alpha}{\pi}} e^{-\alpha\left(\mathbf{r}-\mathbf{r}_{\mathbf{I}}\right)^{2}}
$$

The parameter $\alpha$ is chosen to minimize the total energy. Hence the localized density is

$$
\rho_{e G}(\mathbf{r})=\frac{2 \alpha}{\pi} \sum_{I} e^{-2 \alpha\left(\mathbf{r}-\mathbf{r}_{\mathbf{I}}\right)^{2}}
$$

The gaussian-width parameter $\alpha$ is of the form $a / r_{s}^{3 / 2}$, with $a$ taking a lower-bound value of 0.5 (see Ref [18]). These two forms of the density will be studied below, and the Gaussian approximation will be justified by comparison with results from detailed plane-wave calculations. 
TABLE I: The Madelung energy, $E_{C \text { ou }}$ per electron are given for different values of the Fang-Howard parameter $\beta=b / r_{s}$ for hexagonal(hex), square(sq), rectangular(rec), centered rectangular(cr) lattices defined by their unit vectors $a_{1}: a_{2}$. The $r_{s}$ parameter in the corresponding HIGFET, Eq. 9 is also given. The energies are in units of $1 / r_{s}$. Thus the Madelung energy in Hartrees for a $\delta$-thin hexagonal lattice is $-1.106103 / r_{s}$

\begin{tabular}{lcccc}
\hline \hline HIGFET $r_{s}$ & $\infty$ & 60606 & 60.606 & 0.06060 \\
$(a 1: a 2) \beta \rightarrow$ & 0 & $10^{-2}$ & $10^{-1}$ & 1 \\
\hline $\operatorname{hex}(\sqrt{3}: 1)$ & -1.106103 & -1.052959 & -0.591433 & 3.144793 \\
$\operatorname{cr}(\sqrt{2}: 1)$ & -1.104080 & -1.050937 & -0.589507 & 3.145401 \\
$\operatorname{sq}(1: 1)$ & -1.100244 & -1.047103 & -0.585854 & 3.146555 \\
$\operatorname{rec}(\sqrt{2}: 1)$ & -1.078201 & -1.025072 & -0.564890 & 3.153217 \\
$\operatorname{rec}(\sqrt{3}: 1)$ & -1.042843 & -0.989733 & -0.531301 & 3.163948 \\
\hline \hline
\end{tabular}

\section{B. Calculation with the $\delta$-distribution}

Using Eq. (17) and (21) we have

$$
\begin{aligned}
E_{e e}(q) & =\int_{0}^{\infty} \frac{d y}{2 \sqrt{\pi y}} f(y) \sum_{I \neq J} e^{i \mathbf{q} \cdot\left(\mathbf{r}_{\mathbf{I}}-\mathbf{r}_{\mathbf{J}}\right)} e^{-y\left|\mathbf{r}_{\mathbf{I}}-\mathbf{r}_{\mathbf{J}}\right|^{2}}(24) \\
f(y) & =\int_{0}^{\infty} d z \int_{0}^{\infty} d z^{\prime} \eta(z) \eta\left(z^{\prime}\right) e^{-y\left(z-z^{\prime}\right)^{2}}
\end{aligned}
$$

The details of the evaluation are given in the Appendix.

We have evaluated $E_{C o u}$, Eq. 12 for different lattices: square, rectangular, hexagonal and centered-rectangular. The Coulomb energy depends only on $\beta=\left(b / r_{s}\right)=$ $(3 a) /\left(16 r_{s}\right), r=\left(a_{2} / a_{1}\right)$ and $\left\{\rho_{\mathbf{j}}\right\}$. Our numerical calculations of $E_{\text {Cou }}$ are summarized in Table \ Results for $\beta=10^{-2}$ are at unrealistically low HIGFET densities, but are of formal interest. Results for even smaller values of $\beta$ may be found in Fujiki et al [16, 21]. A comparison with the results of Ref. [16] shows that our results are in agreement when a geometrical term arising from the slight difference in the models is taken into account. (As seen from the details given in the appendix, we have an additive term $N(2 a / 3) / r_{s}{ }^{2}=N(32 b / 9) / r_{s}{ }^{2}$ in our calculation while Fujiki and Geldart have $N(33 b / 8) / r_{s}{ }^{2}$. Agreement is obtained if we replace our term by theirs).

It is seen that the total Coulomb energy increases as $\beta=b / r_{s}$ increases for all cases studied. The hexagonal lattice has the lowest energy for all $\beta$. Moreover, there is no crossing between the different energy curves for any of the lattice structures.

The dependence of the total Coulomb energy of the centered-rectangular lattice and rectangular lattice as a function of the ratio $r=a_{1} / a_{2}$ for the quasi-2D system remains similar to the $\delta$-thin case. Two equivalent minima at $r=\sqrt{3}$ and $1 / \sqrt{3}$ correspond to the hexagonal structure. For the rectangular lattice, the minimum corresponds to $r=1$, i.e., to the square structure. We choose the range $\beta=0.05$ to 0.5 , which corresponds to $r_{s} \sim 0.5$ to $\sim 500$ and fit the Madelung energy of the sta-

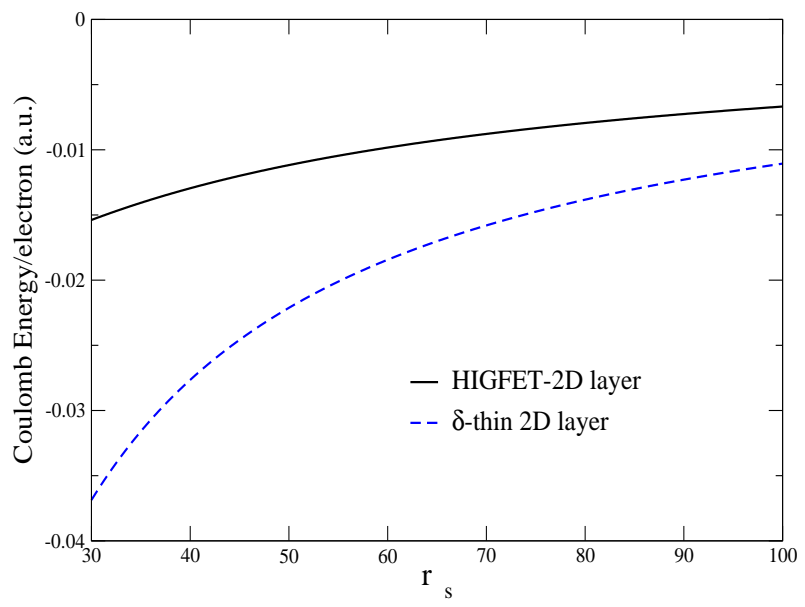

FIG. 2: (Color online) (Coulomb energy per electron in atomic units for a perfectly $2 \mathrm{D}$ system and for $2 \mathrm{D}$ layers in a HIGFET, using Eq.9] to define the thickness.

ble hexagonal lattice (see Table \) to the analytic form

$$
E_{\text {Cou }}\left(r_{s}, \beta\right)=\sum_{i=0}^{i=4} c_{i} \beta^{i} / r_{s}
$$

where $c_{0}=-1.106103, c_{1}=5.34722, c_{2}=-2.15257$, $c_{3}=1.48663$, and $c_{4}=-0.430473$.

In Figure 2 we have plotted the Coulomb energy as a function of $r_{s}$ using Eq. (9) to relate the thickness to the $r_{s}$ value. We observe that the thickness of the system has a significant effect on the energy.

\section{Classical calculation with the gaussian distribution.}

If the electron distribution at each site were a gaussian, the classical electrostatic energy, $E_{e e}$, can be calculated using the same techniques as before (Appendix).

$$
\begin{aligned}
E_{e e}(q, \alpha)= & \frac{1}{2 \sqrt{\pi}} \int_{0}^{\infty} d y y^{-1 / 2}\left(\frac{\alpha}{y+\alpha}\right) e^{-\frac{q^{2}}{4(y+\alpha)}} \\
& \sum_{I \neq J} e^{-\frac{y \alpha}{y+\alpha}\left(\mathbf{r}_{\mathbf{I}}-\mathbf{r}_{\mathbf{J}}\right)^{2}} e^{-i \frac{\alpha}{y+\alpha} \mathbf{q} \cdot\left(\mathbf{r}_{\mathbf{I}}-\mathbf{r}_{\mathbf{J}}\right)}
\end{aligned}
$$

We use the same integral separation with $E_{e e}^{<}$and $E_{e e}^{>}$, the Jacobi function $\theta$ and its transformation. We may verify that when $\alpha$ tends to zero, that is to say the gaussian distribution tends to the $\delta$-distribution, $E_{e e}$ reduces to the Madelung energy of the previous section. Also, if there is no effective overlap among the gaussian distributions, the distributions can be replaced by equivalent point charges at the lattice sites and the Coulomb energy should reduce to the Madelung energy. However, as already remarked by previous authors 18, 19], the charge is not perfectly contained within the Wigner-Seitz disk in 
the $2 \mathrm{D}$ problem. The variations of the thickness and of the lattice type give results similar to the $\delta$-thin case. We consider the variation of $\alpha$ to minimize the total energy within a quantum calculation, and hence do not develop this classical calculation any further.

\section{PERFECTLY TWO-DIMENSIONAL SYSTEMS}

In this section we consider a perfect, i.e., $\delta$ thin 2D layer within a Kohn-Sham density-functional approach 22] to the quantum mechanics of the problem. Since the $\delta$-thin 2D system has been studied extensively, we use it as a reference system to examine the LDA and the CDA as useful tools for calculations of $E_{x c}$ of Wigner crystals. The Hohenberg-Kohn theorem asserts that the total energy is a functional of the one-electron density, and that it is a minimum for the true density distribution. We model the one-electron density as a sum of gaussians centered on each lattice site, and hence the variational problem reduces to a determination of the width parameter $\alpha$ of the Gaussian that minimize the total energy. The total energy of the system at a given $r_{s}$ can be written as:

$$
E_{T}=E_{H F}\left(\alpha, r_{s}\right)+E_{x c}\left(\alpha, r_{s}\right)
$$

where $E_{H F}\left(\alpha, r_{s}\right)$ is the Hartree-Fock energy of an electron. It will be seen that this is effectively the energy of an electron on a single site, and moving in the potential well created by the gaussian distributions on other sites. If the gaussians were perfectly localized, the Coulomb energy would not depend on $\alpha$. The effect of the overlap can be easily included in the variational problem, with the energy given by $\langle\psi|H| \psi\rangle /<\psi \mid \psi>$, and this has an effect for small $r_{s}$. Here $\psi$ is a Slater determinant of gaussians. For the hexagonal lattice, the overlap contribution from two nearest-neighbour gaussians is

$$
s_{i j}\left(r_{s}\right)=\exp \left[-(\alpha / 2)\left(1.09 r_{s}\right)^{2}\right]
$$

where $1.09 r_{s}$ is the nearest-neighbour distance. Unless the contrary is stated, the results reported here will include the overlap correction. The $\alpha$ which minimizes the Hartree-Fock problem is not the same as that which minimized the total energy inclusive of $E_{x c}$. In the next section we look at the problem without $E_{x c}$

\section{A. The Hartree-Fock energy $E_{H F}$}

The Hartree-Fock energy is composed of the classical Madelung energy which defines a constant energy term, plus the quantum mechanical energy associated with the motion of the electron in the field of the other electrons. Since the electrons are strongly localized, especially for large $r_{s}$, a Slater determinant made up of one gaussian function at each lattice site is commonly assumed. The
TABLE II: Comparison of the plane-wave calculation [17] of the HF energies $E_{H F}$ of the $\delta$-thin 2D hexagonal Wigner lattice with the single-Gaussian harmonic lattice energies. $E_{h a r}^{*}$ and $E_{h a r}$ are energies without and with the overlap corrections.

\begin{tabular}{ccccc}
\hline \hline$r_{s} \rightarrow$ & 20 & 30 & 40 & 60 \\
\hline$-E_{H F} \times 10$ & 0.447270 & 0.311642 & 0.239528 & 0.164036 \\
$-E_{\text {har }}^{*} \times 10$ & 0.447155 & 0.311786 & 0.239822 & 0.164530 \\
$-E_{\text {har }} \times 10$ & 0.437058 & 0.308344 & 0.238326 & 0.164113 \\
\hline \hline
\end{tabular}

total energy consists of a kinetic energy term and a potential energy term. These two terms are equal by the virial theorem and hence we only need to evaluate the kinetic energy. Usually, Hartree-Fock energies contain a sizable exchange contribution. However, the localized-gaussian exchange energy is easily shown to be negligible, and we called it the Wigner-exchange energy, $E_{X w c}$.

In Table III we compare our localized-gaussian (harmonic) calculation with the results of the extensive planewave HF calculation by Trail et al 23. The results shown in Table [1] show that the localized single-gaussian model is adequate to describe the Hartree-Fock approximation for this system [24].

Note that our calculation is effectively an "Einstein model" of oscillators, and the kinetic energy is given by,

$$
E_{K}(\alpha)=-\frac{N}{2}<\phi_{I}\left|\nabla_{I}^{2}\right| \phi_{I}>=N \alpha
$$

The gaussian width which minimizes the energy may be fitted by the form $\alpha=0.6263 / r_{s}^{1.57}$.

Since the exchange of electrons actually involves a delocalization process, we believe that the exchange integral evaluated with fixed gaussians does not lead to a true evaluation of the exchange in these systems. The Wigner-exchange energy between two electrons of spin $s_{i}, s_{j}$, is by definition

$$
\begin{aligned}
E_{X w c}^{i j} & =-\int d^{2} r_{i} d^{2} r_{j} \phi_{I}\left(\mathbf{r}_{\mathbf{i}}\right) \phi_{J}\left(\mathbf{r}_{\mathbf{j}}\right) \frac{1}{r_{i j}} \phi_{I}\left(\mathbf{r}_{\mathbf{j}}\right) \phi_{J}\left(\mathbf{r}_{\mathbf{i}}\right) \\
& =-\sqrt{\alpha \pi} e^{-\alpha\left(\mathbf{r}_{\mathbf{I}}-\mathbf{r}_{\mathbf{J}}\right)^{2}} \delta_{s_{i}, s_{j}}
\end{aligned}
$$

We can define a polarization parameter $\zeta=\left(N_{\uparrow}-\right.$ $\left.N_{\downarrow}\right) / N$, therefore

$$
E_{X w c}(\alpha, \zeta)=-\frac{1}{2} \sum_{i \neq j} E_{X w c}^{i j}
$$

This $E_{X w c}$, may be safely neglected for the values of $\alpha$ occurring in this problem.

\section{B. The CDA exchange and correlation energies}

The correlation energy is the most difficult object to calculate, and QMC has been the preferred approach, 
even though this requires a a major numerical effort. However, for an uniform density profile, the correlation energy is well known [3]. Hence, as in Eq.i 2] we map the inhomogeneous density in the $(x, y)$ plane, $\rho(\mathbf{r})$, to a homogeneous form via the $<\rho(\mathbf{r})^{2}>$ average of the CDA method. Given a gaussian distribution,

$$
\begin{aligned}
\bar{\rho} & =\frac{1}{\pi r_{s}^{2}} \int d^{2} r|\phi(\mathbf{r})|^{2}|\phi(\mathbf{r})|^{2} \\
& =\frac{\alpha}{\pi^{2} r_{s}^{2}}
\end{aligned}
$$

We define the effective $r_{s}$ parameter $\overline{r_{s}}$ corresponding to the CDA density by $\bar{\rho}=1 /\left(\pi \bar{r}_{s}^{2}\right)$,

$$
\bar{r}_{s}=r_{s} \sqrt{\frac{\pi}{\alpha}}
$$

The correlation energy in the CDA, $E_{c}^{c d a}$ for the inhomogeneous distribution, inclusive of spin-polarization effects, is now evaluated using $\bar{r}_{s}$ in any of the well-known 2D-functionals [3]. Note that for typical values of $\alpha$ at $r_{s}=20$, the CDA density parameter is $\sim 400$, while at $r_{s}=100$, it becomes $\sim 7000$. Thus we see that the CDA replaces the inhomogeneous fluid with sharp Gaussian peaks by a uniform, ultra-low-density 2D fluid. In calculating $E_{c}\left(\bar{r}_{s}\right)$ using, say, the formula due to Attaccalite et al., a difficulty arises since it is fitted to a maximum $r_{s}$ of 40 , together with asymptotic forms, while the CDA calls for $r_{s}$ values which are one or two orders bigger. Nevertheless, we find surprisingly good results (see below).

At this point we ask if the exchange energy, evaluated for this ultra-low density fluid, should also be included. We believe that this is indeed the case. The fixed-gaussian Wigner-exchange, Eq. 30 simply does not allow any exchange, and ignores the possibilities of tunneling, ring-exchange etc., that exist in the system. We consider that the estimate of exchange obtained from the ultra-low density fluid of the CDA accounts for such exchange effects. This point of view is justified post-facto by the good agreement of our total energies with the QMC total energies.

\section{The LDA exchange and correlation energies}

Another approach to replacing the inhomogeneous electron density by a homogeneous fluid-density is the local-density approximation (LDA) 22]. Here a uniform density corresponding to each local density $n(r)$ is invoked. Thus a local-density parameter $\bar{r}_{s}(r)$ is defined by

$$
\frac{1}{\pi \overline{r s}^{2}}=\frac{\rho(r)}{\pi r_{s}^{2}} \quad \Longrightarrow \quad \bar{r}_{s}(r)=r_{s} e^{\alpha r^{2}} \sqrt{\frac{\pi}{2 \alpha}} .
$$

Hence, knowing the exchange-correlation energy density $e_{x c}$ for a homogeneous system, the exchange-correlation energy of the inhomogeneous system is given by

$$
E_{x c}^{L D A}=\int d^{2} r e_{x c}\left[\bar{r}_{s}(r)\right] \rho(r)
$$

TABLE III: Results of energy minimization for a hexagonal lattice. The Madelung energy $E_{M}=-1.106103 / r_{s}$ has been subtracted out from the total energy. The CDA and LDA results are compared with the GFMC calculations of Tanatar \& Ceperley, T-C [5] The energies are in $10^{-2}$ atomic units.

\begin{tabular}{cccccc}
\hline \hline$r_{s} \rightarrow$ & 20 & 30 & 40 & 50 & 60 \\
\hline CDA & 0.9247 & 0.4824 & 0.3059 & 0.2156 & 0.1625 \\
LDA & 0.9404 & 0.4899 & 0.3102 & 0.2185 & 0.1645 \\
T-C & 0.9167 & 0.4983 & 0.3234 & 0.2313 & 0.1758 \\
\hline \hline
\end{tabular}

Just as in the CDA, the LDA demands the evaluation of $E_{c}$ at densities which are beyond the range of the standard fits. Thus LDA needs $r_{s}(r) \sim 300$ to 5000 at $r_{s}=20$, i.e., a little less extreme than the CDA. Hence, some of the shortcomings of the LDA may also be due to poorly known correlation energies at the exceptionally high $r_{s}$ values that are required. The LDA can be further improved by including gradient corrections. However, we have not included them in this study.

\section{Minimization of the total energy $E_{T}$}

We have now all the energy contributions needed to calculate the ground-state energy of a perfect twodimensional (i.e., $\delta$-thin) Wigner crystal at a given value of the density parameter $r_{s}$. The energy minimum with respect to $\alpha$ is found to be insensitive to the polarization of the lattice. This in agreement with previous studies [3, 4, 5, 25]. In Table III] we give the energy correction to the Madelung energy obtained by the minimization of $E_{T}$, using the CDA or the LDA for evaluating the exchange-correlation effects, together with the results of previous work [5]. QMC results by Rapisarda and Senatore [4] are very similar to those of Tanatar et al., and the agreement is similar. The optimal $\alpha$ which minimizes the energy is found to be given by $\alpha=a / r_{s}^{3 / 2}$ with $\mathrm{a}=0.639$ for both CDA and LDA approaches. Here we note that Nagy's method [18] predicts a value $\alpha_{n g}=0.5 / r_{s}^{3 / 2}$ as a lower bound. A crucial test of the accuracy of the CDA and LDA would lie in their ability to predict the liquid $\rightarrow$ solid phase transition. This is addressed in section IVC The total energy can be represented by:

$$
E_{T}\left(r_{s}\right)=\frac{a_{1}}{r_{s}}+\frac{a_{2}}{r_{s}^{3 / 2}}+\frac{a_{3}}{r_{s}^{2}}+O\left(r_{s}^{-5 / 2}\right) \quad r_{s} \gg 1
$$

where $a_{1}=-1.106103$ is the Madelung constant and $a_{2}$ is the zero-point energy of the lattice. We determined the coefficient $a_{3}$ by a least-square fit. The results are summarized in Table IV together with previous results. These results justify our use of the CDA and the LDA for evaluating the total energy of quasi-2D Wigner crystal phases for which there are no QMC calculations as yet. 
TABLE IV: Coefficients $a_{1}-a_{3}$ in Eq. 35fitting the CDA and LDA total energy (for the range $r_{s}=20$ to 100) are compared with previous work.

\begin{tabular}{cccccc}
\hline \hline & CDA & LDA & BM[7] & RS[4] & TC[5] \\
\hline$-a_{1}$ & 1.1061 & 1.10610 & 1.1060 & 1.104715 & 1.10610 \\
$a_{2}$ & 0.8142 & 0.8142 & 0.8142 & 0.7947 & 0.8142 \\
$a_{3}$ & 0.2456 & 0.1194 & $\ldots$ & 0.07338 & 0.0254 \\
\hline \hline
\end{tabular}

\section{INFLUENCE OF THE THICKNESS}

We consider a quasi-2D electron crystal where each electron is localized at each lattice site with a gaussian distribution centered on each site in the $(x, y)$-plane, while the $z$-extension may typically have the form of a Fang-Howard density. As before, such z-distributions can be replaced by a constant-density form for ease of calculations. Also, we assume that the 2D layers are in HIGFETS, and as such the FH-parameter $b$ is automatically specified (via Eq. 9])when the $r_{s}$ parameter defining the 2D-layer density is specified.

The kinetic energy and the harmonic energy of the quasi-2D system are still given by $E_{K}(\alpha)=N \alpha$ since this is a result of the assumed gaussian form of the wavefunction. However, the simple Coulomb potential $1 / r$ has changed to $F(r) / r$ where $F(r)$ is the form factor arising from the subband distribution. The Wigner-exchange energy, i.e., the exchange between two localized electrons is now even weaker than in Eq. 29] Hence this type of exchange is totally negligible.

\section{A. The evaluation of $E_{x c}$ for thick-2D layers using CDA and LDA.}

As described in Eq. 9] the z-distribution is mapped onto a uniform slab of width $a$; in HIGFETS this is directly related to the $r_{s}$ parameter in the 2-D plane. The inhomogeneous 2-D distribution in the plane is also mapped onto a homogeneous distribution via the CDA as in Eqs. 32 or as in Eq. 33 for the LDA. Both CDA and LDA require a knowledge of the $E_{x c}\left(r_{s}, a\right)$ for quasi$2 \mathrm{D}$ uniform systems with a layer width $a$. Parametrized forms of the exchange energy and the correlation energy of uniform quasi-2D electron fluids have been given by Dharma-wardana 10]. The exchange energy $E_{x}\left(r_{s}, a\right)$ is given in the form $E_{x}\left(r_{s}, \zeta\right) Q\left(r_{s}, a, \zeta\right)$ where $E_{x}\left(r_{s}, \zeta\right)$ is the well-known exchange energy of the $\delta$-thin system, while $Q\left(r_{s}, a, \zeta\right)$ is a form factor. The correlation energy of quasi-2D layers in HIGFETS is given in Ref. [10] as an interpolation involving a form for electron "rods" interacting via a logarithmic potential (as is the case for small $r_{s}$ ), and for 3D-like electrons when $r_{s}$, and the thickness $a$ become large. The Wigner crystallization regime involves the latter regime. For details of these parametrizations, the reader is referred to Ref. [10]. Since the WT involves

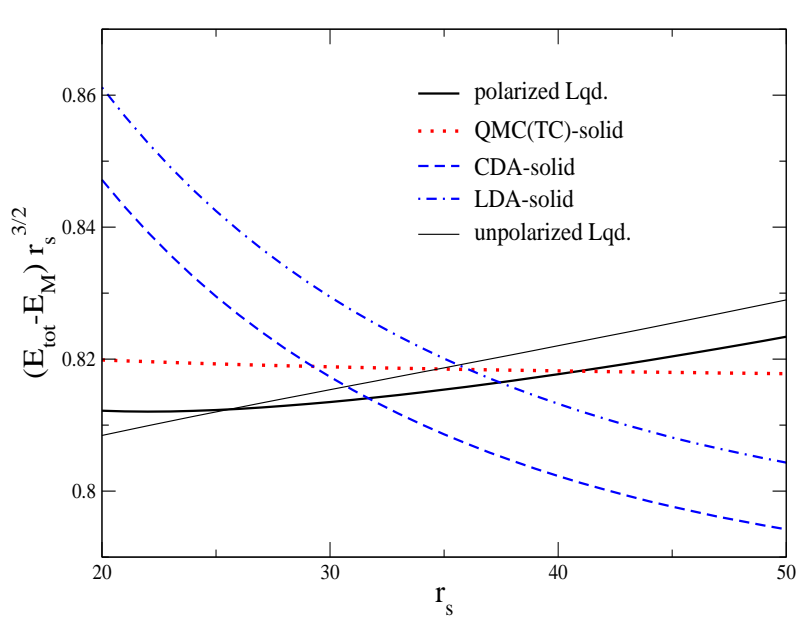

FIG. 3: (Color online) Comparison of liquid and solid-phase energies. $\left(E-E_{M}\right) r_{s}{ }^{3 / 2}$ where $E_{M}=-1.106103 / r_{s}$ and $E$ is the unpolarized or fully-polarized fluid energy, or the solid energy $E_{c d a}, E_{l d a}$ or from QMC.

small energy differences, we have actually done an explicit calculation instead of using the fits.

\section{B. Minimization of the total energy $E_{T}$}

As in Sec IID we minimize the total energy as a function of $\alpha$ for a given $r_{s}$. Here, the energy is more sensitive to the spin-polarization $\zeta$ than in the perfect crystal even if the difference is very small. The unpolarized crystal is more stable than the polarized one. So we present results for the unpolarized system. The values of $\alpha$ which minimizes the energy can also be fitted by the same form as in SecIIID We obtain

$$
\alpha_{c d a}=\frac{0.619}{r_{s}^{3 / 2}} \quad \text { and } \quad \alpha_{l d a}=\frac{0.627}{r_{s}^{3 / 2}}
$$

We have also fitted the total energy. Here the Madelung energy is the $E_{C o u}$ given in Eq.(25) and we use the usual expansion in inverse $r_{s}^{3 / 2}$ etc.

$$
\begin{aligned}
& E_{T}^{c d a}=E_{\text {Cou }}\left(r_{s}\right)+\frac{0.68597}{r_{s}^{3 / 2}}+\frac{0.321652}{r_{s}^{2}} \\
& E_{T}^{l d a}=E_{\text {Cou }}\left(r_{s}\right)+\frac{0.708977}{r_{s}^{3 / 2}}+\frac{0.357242}{r_{s}^{2}}
\end{aligned}
$$

We remark that the total energy has a minimum as a function of $r_{s}$. This minimum is located around $r_{s} \sim 26$ and its value is $\sim-0.011$ a.u. A comparison of liquid phase and Wigner crystal in HIGFETS is given in Table $\nabla$ These total energies include the $2 a / 3 r_{s}^{2}$ correction arsing from the interaction of the quasi-2D layer with the unifrom background, as discussed in subsection IIB Since this depends on the layer thickness $a$, this correction does not occur in the ideal $2 \mathrm{D}$ system. 
TABLE V: Results of energy minimization for a hexagonal lattice and comparison with the unpolarized liquid phase energy $E_{L}$. The energies are measured in $10^{-3}$ atomic units.

\begin{tabular}{ccccc}
\hline \hline$r_{s}$ & 15 & 20 & 30 & 50 \\
\hline$E_{c d a}$ & -6.7255 & -10.1581 & -10.8576 & -9.1112 \\
$E_{l d a}$ & -6.5036 & -9.8169 & -10.7782 & -9.0306 \\
$E_{L}$ & -7.1324 & -10.0249 & -10.5995 & -8.8939 \\
\hline \hline
\end{tabular}

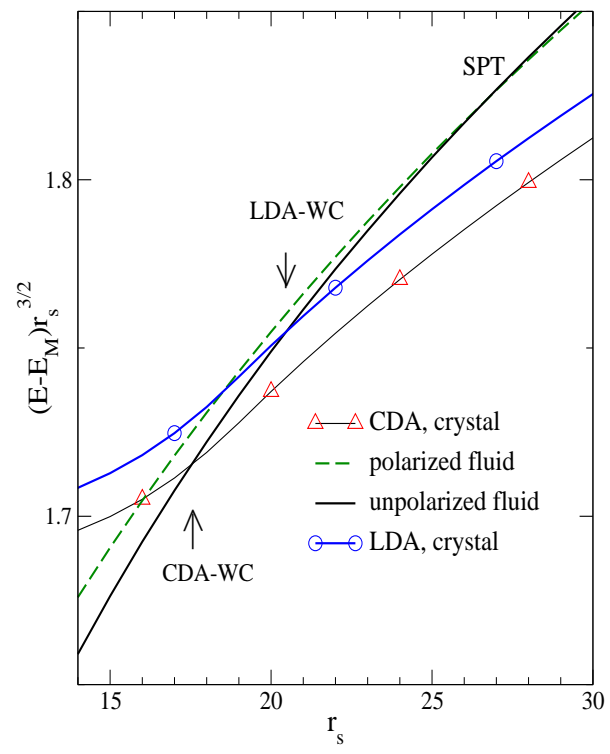

FIG. 4: (Color online) $\left(E-E_{M}\right) r_{s}{ }^{3 / 2}$ where $E_{M}=$ $-1.106103 / r_{s}$ and $E$ is the unpolarized or fully-polarized fluid energy, or the solid energy $E_{c d a}$ or $E_{l d a}$. The spin-phase transition in the fluid is labeled SPT. The onset of the Wigner crystal in CDA and LDA are indicated by arrows.

\section{Phase transition Liquid $\rightarrow$ Wigner crystal.}

According to quantum Monte Carlo simulations, the phase transition between a $\delta$-thin $2 \mathrm{D}$ electron liquid and a 2D electron Wigner crystal occurs around $r_{s}=35 \pm 5$. With our methods we find a transition for $r_{s}=32$ using the CDA and $r_{s}=38$ using the LDA.

In Figure 31 we show the phase diagram of the system (in order to have a clear display we present $\left(E-E_{M}\right) r_{s}{ }^{3 / 2}$ where $E_{M}=-1.106103 / r_{s}$ the Madelung energy). The fluid phase energy is calculated using the fit given by 3 . Unlike in the $\delta$-thin 2D system, the total energy contains the term $\Delta_{b e}=2 a / 3 r_{s}^{2}$ arising from the interaction with the unifrom background, (see subsection [IB). This has been removed from both the liquid and the solid phase energies as this improves the clarity of the display.

These results tend to show that both LDA and CDA provide an adequate evaluation of $E_{x c}$ for electron solids, especially when we recall that the $E_{c}$ at $r_{s}$ values (200$8000)$, way outside the fit range $\left(r_{s} \leq 40\right)$, are needed in the CDA and the LDA evaluations. Figure 4 displays the phase transitions in the quasi-2D HIGFET system. The
Wigner transition occurs at $r_{s}=15.5$ for the CDA, and $r_{s}=20.5$ for the LDA, i.e, before the spin-phase transition (marked SPT in the figure) of the liquid phase. Since the $\delta$-thin 2D layer is expected to have a WT near $r_{s} \sim 35$, the thickness effect has brought the WT to smaller $r_{s}$ values. It should be noted that if correlation effects are neglected, the WT occurs at very low $r_{s}$. Hence the shift of the WT to low $r_{s}$ is a consequence of the reduced correlations in the quasi-2D system.

\section{CONCLUSION}

We have investigated the crystallization of electrons under their own interaction in infinitely-thin 2D electron layers, known as Wigner crystallization, as well as in 2D layers with a finite width. The case of infinitely-thin 2D electron layers has been extensively studied in the past, and provides a bench mark to test our methods for replacing inhomogeneous electron densities by equivalent uniform-density models. Detailed Hartree-Fock calculations with large plane-wave basis sets are shown to be closely equivalent to the single-gaussian harmonic lattice calculations. We show that the constant-density approximation, CDA, as well as the local-density approximation, LDA, successfully recover the total energy as well as the correlation energies of the infinitely-thin 2D electron crystal. In particular, these models predict a liquid $\rightarrow$ solid phase-transition in the range $30<r_{s}<40$, in good agreement with Quantum Monte Carlo simulations. When these methods are applied to quasi-2D layers with the thickness as in HIGFETS, the weakened Coulomb correlations move the Wigner transition towards high densities. The LDA and the CDA predict a Wigner transition near $r_{s} \sim 15$ to 21 .

\section{APPENDIX: EVALUATION OF THE ELECTROSTATIC ENERGY AND LATTICE SUMS.}

The expression for the electron-electron Coulomb energy, Eq. 24] can be rewritten, using the $\theta$ Jacobi function technique as,

$$
\begin{aligned}
E_{e e}(q)= & \frac{N}{2 \sqrt{\pi}} \sum_{j} \int_{0}^{\infty} d y y^{-1 / 2} f(y) e^{-y\left|\rho_{\mathbf{j}}\right|^{2}-i \mathbf{q} \cdot \rho_{\mathbf{j}}} \\
& \left(\prod_{\alpha} \theta\left[\frac{a_{\alpha}}{2 \pi}\left(2 \rho_{j}^{\alpha} y+i q_{\alpha}\right) ; \frac{y a_{\alpha}{ }^{2}}{\pi}\right]-\delta_{i, 0}\right)
\end{aligned}
$$

where $\delta_{i, 0}$ is the Kronecker symbol, because when $\rho_{\mathbf{j}}=0$, we must have $(m, n) \neq\left(m^{\prime}, n^{\prime}\right)$.

The advantage of introducing the Jacobi function $\theta(z, X)$ is that it converges well for large $X$ and we are also able to obtain convenient well-convergent formulas for the small$\mathrm{X}$ region by applying the transformation (Eq. (20) from which the Coulomb singular part at $q \rightarrow 0$ can be rigorously extracted. Thus, $E_{e e}(q)$ obtained in Eq.A.1 can 
be separated into a large $y$ part and a small $y$ part given where by

$$
E_{e e}(q)=E_{e e}^{>}(q)+E_{e e}^{<}(q)
$$

$$
E_{e e}^{>}(q)=\frac{N}{2 \sqrt{\pi}} \sum_{j} \int_{y_{0}}^{\infty} d y y^{-1 / 2} f(y) e^{-y\left|\rho_{\mathbf{j}}\right|^{2}-i \mathbf{q} \cdot \rho_{\mathbf{j}}}\left(\prod_{\alpha=x, y} \theta\left[\frac{a_{\alpha}}{2 \pi}\left(2 \rho_{j}^{\alpha} y+i q_{\alpha}\right) ; \frac{y a_{\alpha}{ }^{2}}{\pi}\right]-\delta_{i, 0}\right)
$$

and

$$
\begin{aligned}
E_{e e}^{<}(q)= & \frac{N}{2 \sqrt{\pi}} \sum_{j} \int_{0}^{y_{0}} d y y^{-1 / 2} f(y) e^{-y\left|\rho_{\mathbf{j}}\right|^{2}-i \mathbf{q} \cdot \rho_{\mathbf{j}}}\left(\prod_{\alpha=x, y} \theta\left[\frac{a_{\alpha}}{2 \pi}\left(2 \rho_{j}^{\alpha} y+i q_{\alpha}\right) ; \frac{y a_{\alpha}{ }^{2}}{\pi}\right]-\delta_{i, 0}\right) \\
= & \frac{N \sqrt{\pi}}{2 a_{1} a_{2}} \sum_{j} \int_{0}^{y_{0}} d y y^{-3 / 2} f(y) e^{-\frac{q^{2}}{4 y}}\left(\prod_{\alpha=x, y} \theta\left[-i \frac{2 \rho_{j}^{\alpha} y+i q_{\alpha}}{2 a_{\alpha} y} ; \frac{\pi}{y a_{\alpha}{ }^{2}}\right]-1+1\right) \\
& -\frac{N}{2 \sqrt{\pi}} \int_{0}^{y_{0}} d y y^{-1 / 2} f(y) \\
= & \frac{N \sqrt{\pi}}{2 a_{1} a_{2}} \sum_{j} \int_{0}^{y_{0}} d y y^{-3 / 2} f(y) e^{-\frac{q^{2}}{4 y}}\left(\prod_{\alpha=x, y} \theta\left[-i \frac{2 \rho_{j}^{\alpha} y+i q_{\alpha}}{2 a_{\alpha} y} ; \frac{\pi}{y a_{\alpha}{ }^{2}}\right]-1\right) \\
& -\frac{n_{l} N \sqrt{\pi}}{2 a_{1} a_{2}} \int_{y_{0}}^{\infty} d y y^{-3 / 2} f(y) e^{-\frac{q^{2}}{4 y}}-\frac{N}{2 \sqrt{\pi}} \int_{0}^{y_{0}} d y y^{-1 / 2} f(y)+E_{e e}^{h o m}(q)
\end{aligned}
$$

where $n_{l}$ is the number of sublattices $\left(a_{1} a_{2} / n_{l}=\pi r_{s}{ }^{2}\right)$. The results of the calculation are independent of the value of $y_{0}>0$; nevertheless, we choose it such that the sums $\theta$ converge fast, and we have

$$
E_{e e}^{h o m}(q)=\frac{n_{l} N \sqrt{\pi}}{2 a_{1} a_{2}} \int_{0}^{\infty} d y y^{-3 / 2} f(y) e^{-\frac{q^{2}}{4 y}}
$$

In order to complete the calculation of $E_{C o u}$, we need to discuss the form of $\eta(z)$. In their article [16], Fujiki and Geldart use the Fang-Howard density $\eta_{f h}(z)=\frac{1}{2 b^{3}} z^{2} e^{-\frac{z}{b}}$ (Figure 11). As already discussed we replace the FH distribution by the equivalent $\mathrm{CDA}$, i.e., we use a constant density slab of width $a=16 b / 3$. With this homogeneous form of density

$$
\begin{aligned}
f(y) & =\bar{\eta}^{2} \int_{0}^{a} d z \int_{0}^{a} d z^{\prime} e^{-y\left(z-z^{\prime}\right)^{2}} \\
& =\bar{\eta}^{2} \frac{\left(e^{-a^{2} y}+a \sqrt{\pi y} \operatorname{erf}(a \sqrt{y})-1\right)}{y}
\end{aligned}
$$

We see here an advantage of the constant density mapping to density $\bar{\eta}$, the analytic expression of $f(y)$ being quite simple.
In Eq.(18), we replace $\eta\left(z^{\prime}\right)$ by its expression

$$
\begin{aligned}
E_{e d}(q) & =-\frac{N}{q r_{s}^{2}} \frac{e^{-q b_{d}}}{a q}\left(1-e^{-a q}\right) \\
E_{e d}(q \rightarrow 0) & =-\frac{N}{r_{s}^{2}}\left(\frac{1}{q}-\frac{a}{2}-b_{d}+O(q)\right)
\end{aligned}
$$

We recall that $b_{d}$ is positive or zero, and gives the location of the donor layer at $z=-b_{d}$. Now, in Eq. A.4, we use the definition of $f(y)$.

$$
\begin{aligned}
E_{e e}^{h o m}(q) & =\frac{N}{q r_{s}^{2}} \frac{2}{q a^{2}}\left(a-\frac{1}{q}\left(1-e^{-a q}\right)\right) \\
E_{e e}^{h o m}(q \rightarrow 0) & =\frac{N}{r_{s}^{2}}\left(\frac{1}{q}-\frac{a}{3}+O(q)\right)
\end{aligned}
$$

Now, we will use Eq.(16), (A.6), A.2), A.3), A.7) in Eq. (12) to calculate the Coulomb energy for different types of lattices and for different thicknesses. We can see that the expression of $E_{C o u}$ is dependent on the parameter $b_{d}$. Since this is a constant contribution, we set $b_{d}=0$ and focus on the part which depends only on the geometry of the lattice and on its thickness.
[1] T. Ando, A. B. Fowler, F. Stern, Rev. Mod. Phys. 54, $437(1982)$
[2] S. V. Kravchenko and M. P. Sarachik, Rep. Prog. Phys. 
67, 1 (2004)

[3] C. Attaccalite, S. Moroni, P. Gori-Giorgi, G. B. Bachelet, Phys. Rev. Lett 88, 256601(2002)

[4] F. Rapisarda, G. Senatore, Aust. J. Phys. 49, 161(1996)

[5] B. Tanatar, D. M. Ceperley, Phys. Rev. B 39, 5005 (1989)

[6] D. Ceperley, Phys. Rev. B 18, 3126 (1978)

[7] L. Bonsall, A. A. Maradudin, Phys. Rev. B 15, 1959 (1977)

[8] R. Rajeswara Palanichamy and K. Iyakutti, Int. J. Quant. Chem. 102, 112 (2005)

[9] François Perrot and M. W. C. Dharma-wardana, Phys. Rev. Lett. 87, 206404 (2001)

[10] M. W. C. Dharma-wardana, Solid State Com. 136, 76 (2005); cond-mat/503246; cond-mat/506816.

[11] Y.-W Tan, J. Zhu, H. L. Stormer, L. N. Pfeiffer, K. N. Baldwin, and K. W. West, cond-mat/0412260 Phys. Rev. Lett. 94, 16405, (2005).

[12] J. Zhu, H. L. Stormer, L. N. Pfeiffer, K. W. Baldwin, and K. W. West, Phys. Rev. Lett. 90, 56805 (2003)

[13] R. Asgari, B. Davoudi, M. Polini, M. P. Tosi, G. F. Giuliani, and G. Vignale, Phys. Rev. B 71, 45323 (2005); cond-mat/0412665

[14] Y. Zhang and S. Das Sarma, Phys. Rev. B 71,45322 (2005); cond-mat/0312565

[15] S. De Palo, M. Botti, S. Moroni, and G. Senatore, Phys. Rev. Lett. 94, 226405 (2005); cond-mat/0410145
[16] N. M. Fujiki, D. J. W. Geldart, Phys. Rev. B 46, 9634(1992)

[17] J. R. Trail, M. D. Towler, R. J. Needs, Phys. Rev. B 68, 045107 (2003)

[18] I. Nagy, Phys. Rev. B 60, 4404 (1999)

[19] M. Polini, G. Sica, B. Davoudi, M. P. Tosi, J. Phys. Cond. Mat. 13, 3591 (2001)

[20] P. Gori-Giorgi and A. Savin, Phys. Rev. A 7132513 (2005); cond-mat/0411179

[21] N. M. Fujiki, K. De'Bell, D. J. W. Geldart, Phys. Rev. B 36, 8512 (1987)

[22] W. Kohn, P. Vashishta, iTheory of the inhomogeneous electron gas, edited by S. Lundqvist and N. H. March, Plenum Press (1983)

[23] We thank Dr. Trail et al., for kindly supplying us their tabulation of the Hartree-Fock energy of the 2D wigner crystal as a function of $r_{s}$.

[24] N. D. Drummond, Z. Radnai, J. R. Trail, M. D. Towler, and R. J. Needs, Phys. Rev. B 69, 85116 (2004); Drummond et al. have come to a similar conclusion about the Hartree-Fock energy of the 3D-Wigner crystal.

[25] X. Zhu, S. G. Louie, Phys. Rev. B 52, 5863 (1995)

[26] P. Gori-Giorgi, J. P. Perdew, Phys. Rev. B 69, 041103 (2004)

[27] J. P. Perdew, Y. Wang, Phys. Rev. B 45, 13244 (1992) 